\title{
Kernos
}

Revue internationale et pluridisciplinaire de religion grecque antique

$25 \mid 2012$

Varia

\section{Richard VEYMIERS, "Hileôs tôi phorounti". Sérapis sur les gemmes et les bijoux antiques}

\author{
Laetizia Puccio
}

\section{OpenEdition}

Journals

\section{Édition électronique}

URL : http://journals.openedition.org/kernos/2071

DOI : 10.4000/kernos.2071

ISSN : 2034-7871

\section{Éditeur}

Centre international d'étude de la religion grecque antique

\section{Édition imprimée}

Date de publication : 26 octobre 2012

Pagination : 368-369

ISSN : 0776-3824

Référence électronique

Laetizia Puccio, "Richard veymiers, "Hileôs tôi phorounti". Sérapis sur les gemmes et les bijoux antiques », Kernos [En ligne], 25 | 2012, mis en ligne le 01 octobre 2012, consulté le 21 septembre 2020. URL : http://journals.openedition.org/kernos/2071 ; DOI : https://doi.org/10.4000/kernos.2071

Ce document a été généré automatiquement le 21 septembre 2020.

Kernos 


\title{
Richard VEYMIERS, "Hileôs tôi phorounti". Sérapis sur les gemmes et les bijoux antiques
}

\author{
Laetizia Puccio
}

\section{RÉFÉRENCE}

Richard VEYMIERS, "Hileôs tôi phorounti". Sérapis sur les gemmes et les bijoux antiques, Bruxelles, Académie royale de Belgique, 2009. 1 vol. 22,5 × 28,5 cm, 608 p. (Classe des Lettres). ISBN : 978-2-8031-0261-7.

1 Au sein de l'histoire des religions antiques, les études isiaques se caractérisent par une interdisciplinarité qui n'est plus à démontrer et qui ouvre une série de perspectives renouvelées ${ }^{1}$. Parmi la masse documentaire à laquelle sont confrontés les chercheurs, R. Veymiers (R.V.) s'est attaqué avec rigueur et passion à un type de sources iconographiques souvent sous-estimé, celui des gemmes et des bijoux. Son étude s'est attachée à la figure de Sérapis, un dieu considéré dès l'origine, dans l'Égypte des premiers lagides, comme le parèdre d'Isis.

2 Après une brève introduction sur la création, l'identité et le succès de Sérapis ainsi que sur les difficultés et les intérêts de la documentation, l'A. a divisé son travail en deux parties. Dans la première, il présente une étude historique des différentes représentations du dieu. Le texte est accompagné des exemples qui constituent la seconde partie du travail, à savoir le catalogue. Les deux volets de l'enquête reprennent la même typologie : les bustes et les têtes de Sérapis ; Sérapis trônant; Sérapis debout; Sérapis allongé sur la klinè; Sérapis accompagné d'autres divinités; Sérapis assimilé à d'autres divinités. Le chercheur a réuni 1259 objets - dont de nombreux inédits - qu'il a organisés en 74 types (tableau p. 220-221). Chaque document est identifié selon la nature du support, le lieu de provenance, la datation, le matériau, les dimensions, le 
lieu de conservation, le renvoi à l'illustration, la description du motif, les références bibliographiques, les éventuelles copies.

3 La majorité des pièces ( $75 \%$ ) figurent le dieu en buste, tandis qu'il se présente également trônant et en pied, parfois même allongé sur la klinè ou en Agathos Daimon. Neuf fois sur dix, Sérapis porte le calathos, alors que l'atef n'apparaît qu'à 49 reprises. Parmi ses attributs, le sceptre et Cerbère sont les plus courants, mais l'aigle, la patère, le foudre, la couronne ou la cornucopia sont également attestés, tout comme d'autres animaux, tels le griffon et le bélier, et d'autres accessoires, tels le zodiaque, les planètes, la lune, les étoiles ou les enseignes militaires. Lorsqu'il est accompagné d'une autre divinité, c'est Isis qui se trouve le plus fréquemment à ses côtés, tandis qu'Harpocrate n'est associé que deux fois à son père. Dans les triades, les groupes Sérapis et les Nikè ainsi que Sérapis et les Dioscures l'emportent sur ceux d'Isis-SérapisHarpocrate et Isis-Sérapis-Déméter. Alors qu'Anubis ne forme jamais de duo avec le dieu alexandrin, il se tient auprès de lui et de son épouse une seule fois. Deux exemplaires uniquement montrent la tétrade Sérapis-Isis-Harpocrate-Anubis. Il est possible de rencontrer auprès de lui d'autres divinités comme Aphrodite, Apollon, Artémis, Asclépios, Athéna, Éros, Hélios, Héraclès, Hermès, Horus, Hygie, Jupiter Héliopolitain, Némésis, Séléné, Thot, Tyché-Fortuna et Zeus. Enfin, Sérapis peut être figuré auprès d'une reine d'Égypte, d'un empereur ou d'un membre de l'armée.

Selon les mots de l'A. "si une même fonction peut se traduire par plusieurs schémas iconographiques, une même image peut faire l'objet de diverses lectures » (p. 213). Les multiples apparences de Sérapis sont le reflet de l'enrichissement de sa personnalité. Dieu agraire, souverain des mers, guérisseur, invincible et victorieux, il peut également devenir un dieu à la fois unique et panthée. Les quelques inscriptions gravées dans la pierre, quand elles ne nous donnent pas des renseignements sur le nom de leur propriétaire, traduisent par des formules d'exaltation la toute-puissance du dieu. Dans $5 \%$ des cas, la signification de ces objets concerne le monde de la magie.

5 R.V. situe la fabrication des gemmes et des bijoux à l'image de Sérapis d'abord en Égypte, notamment dans les ateliers alexandrins. Les contextes de découverte lorsqu'ils sont connus ( $20 \%$ de la documentation) - témoignent des différents modes d'utilisation de ces pièces. Elles pouvaient être considérées à la fois comme des objets de parure, des sceaux, des souvenirs, mais aussi des marqueurs d'identité, des signes de fidélité envers un souverain ou, principalement, des formes de dévotion. Bien que plusieurs images aient été copiées des émissions monétaires, en particulier de celles de l'atelier d'Alexandrie, les artisans n'étaient pas contraints de suivre des règles officielles et ils ont ainsi multiplié les différentes représentations du dieu afin de s'adapter à une clientèle évoluant dans la sphère privée des croyances. Les matières employées sont tout aussi nombreuses : pierres précieuses ou semi-précieuses, pâtes de verre, métaux et terres cuites. Leur diffusion semble avoir débuté durant l'époque hellénistique en Méditerranée orientale pour se répandre ensuite à tout l'Empire.

Le catalogue, directement suivi de la typologie mise en place par l'A., est enrichi de plus de cent pages de planches en noir et blanc ainsi qu'en couleurs. Pour les $20 \%$ de gemmes et bijoux de provenance connue, plusieurs cartes de grande qualité, établies par Fabrice Delrieux, nous montrent leur répartition dans l'ensemble du monde grécoromain, voire au-delà des frontières de l'Empire. Le travail est supporté par une bibliographie de qualité qui témoigne de la maîtrise du sujet par son A. Il est 
accompagné de plusieurs index qui facilitent grandement l'utilisation de ce très beau volume.

7 À travers ce travail, à bien des égards exemplaire, R.V. a démontré combien les gemmes et les bijoux à l'effigie de Sérapis sont un support inestimable pour saisir l'iconographie du dieu, également mise en valeur sur les lampes² ${ }^{2}$ On apprécie en particulier la minutie des descriptions et la prudence dont il a fait preuve dans ses réflexions sur des thèmes aussi complexes que l'iconographie et l'histoire des religions antiques. Le chercheur ne s'est pas arrêté en si bon chemin puisqu'un premier supplément de 121 pièces a déjà été publié ${ }^{3}$ et un second est en cours de réalisation. Il reste à présent à espérer que les autres divinités isiaques bénéficieront un jour, dans le domaine de la glyptique, d'études aussi richement documentées et illustrées.

\section{NOTES}

1. Pour consulter l'ensemble de ces perspectives : L. BRICAULT, «Études isiaques : perspectives ", in L. BRICAUlt (éd.), De Memphis à Rome. Actes du I ${ }^{e r}$ Colloque international sur les études isiaques. Poitiers - Futuroscope, 8-10 avril 1999, Leyde, 2000, p. 189-210; id., «Bilan et perspective des études isiaques », in E. LEOSPO, D. TAVERNA (éd.), La Granda Dea tra passato e presente, Turin, 2000, p. 91-96 ; L. BRICAULT, R. VEYMIERS, « Quinze ans après » (à paraître).

2. Consulter le recueil de lampes isiaques établi récemment par J.-L. PODVIN, Luminaire et cultes isiaques, Montagnac, Monographie Instrumentum 38, 2011.

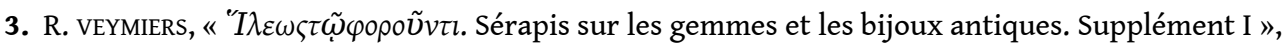
in L. BRICAULT, R. VEYMIERS (éd.), Bibliotheca Isiaca II, Bordeaux, Ausonius Éditions, 2011, p. 239-271.

\section{AUTEURS}

\section{LAETIZIA PUCCIO}

Université de Liège 